\title{
Surveying Through Solid Walls
}

\author{
William C. Stone \\ National Institute of Standards and Technology \\ Gaithersburg, Maryland 20899 \\ william.stone@nist.gov
}

\begin{abstract}
A good metrology system in the automated environment must (1) measure the three dimensional position and attitude of any component to a reasonable degree of accuracy; (2) acquire these data fairly rapidly; and (3) be capable of making reliable measurements anywhere on site. A significant limitation for existing surveying systems (including GPS and laser based technologies) is the requirement for direct line-of-sight between the reference and roving surveying elements. Construction sites, as a rule, are highly unstructured environments and clutter is the norm. Recent fundamental research has been undertaken at NIST to develop a real-time Non-Line-of-Sight measurement system capable of tracking to $10 \mathrm{~mm}$ accuracy despite the presence of walls, ceilings, floors, and other obstructions. The new system determines the distance from a series of reference transmitters which broadcast pulse-synthesized baseband electromagnetic signals. Digital signal processing techniques are used to recover the time of flight (and hence distance) and to compensate for errors introduced as a result of material penetration and retransmission of the signal. Results of the most recent series of NIST laboratory investigations leading towards a practical construction metrology system are discussed.
\end{abstract}

\section{1: Introduction}

The NIST program in Construction Automation seeks to develop, integrate, and implement new technologies which will permit generalized automation at the construction job site. Research is presently focused on methods and standards for closing the information loop from the job site to a central dynamic, evolving project database, and returning information from that database in an on-demand, real-time format to a wide variety of users at the construction site. Current research includes the development of a real-time, nonline-of-sight surveying system that can "see through walls;" the development of the National Construction Automation Test Bed for testing various automation mechanisms,standards, and software; the development of kinematic representation standards for construction machinery and components; and the development of standards for wireless data telemetry packets for transmission of information from the active job site to a high bandwidth trunk line which links the various project participants.

Each of the above research areas leverage upon the rapid advance of information technology and infrastructure that has occurred during the last decade and is still continuing. The emergence of high speed computer communication networks (the "information superhighway") and real-time, immersive, computer graphics (virtual reality) technologies presage the imminent ability to manage remote construction sites from central offices; to automate certain portions of the tasks performed by common construction machines; and to provide information on the state of such machines to operators (on-site or remote) that would greatly enhance their productivity. Limited demonstrations of this type of technology, largely relating to the control of robotic spacecraft and, more pertinently, to the tele-operation of simple machines for the handling of nuclear waste, have been conducted.

In order to achieve acceptance in the construction industry, a standard means of rapidly interfacing any piece of machinery to a construction-site database must be developed. The development of this standard is highly unlikely to be addressed by the construction industry itself, where corporate research budgets are small in the United States. Nor will it be developed by equipment manufacturers who, left to their own devices, tend to develop closed, proprietary systems which by their very nature inhibit the free exchange of data with other systems that might be operational at a construction site. In this respect, NIST has been uniquely positioned, as a neutral entity, to establish the framework for the integration of construction machinery into emerging global database standards. The underpinning to the above technology is the ability to know the real-time position of any piece of equipment and component on the construction site. Present sur- 
veying tools suffer from many shortcomings in this regard, the most important of which is that they must operate under line-of-sight conditions. The development of a non-line-of-sight surveying system, which can in effect "see through walls", represents the pinnacle of construction site metrology and has guided the focus of NIST research in Non-Line-of-Sight (NLS) metrology.

Tests conducted at NIST from 1994 through 1997 proved the potential of NLS technology to meet the above objectives $[1,2,3]$. If the technology can be brought to practical implementation the implications are profound. Briefly summarized, the technology involves the use of ultra-wide bandwidth spread spectrum radio signals which are beamed through nonmetallic construction materials as a series of sequential, discrete frequencies. The same approach applies equally well to an impulse baseband transmission. A timedomain response is reconstructed from the frequency power spectrum and the time of flight from transmitter to receiver is calculated by comparing the measured response to a free-space calibration between two or more known points. Problems arise, however, due to non-linear propagation velocities when penetrating solid media. This leads to a delayed arrival of the straight-line (true distance) transmission pulse, thereby producing error in the calculated range.

Other errors arise from scattering (dispersion) of the beam, refraction, and multi-path reflections as well as instrumental error. However, it was demonstrated, by means of qualitative tests in 1994 [1] and by extensive quantitative tests in 1996 [2], that most common construction materials behave as non-conductors and can therefore be successfully penetrated for substantial distances, thereby allowing range measurement. To be more emphatic, unless the building is constructed with seamless metal walls distance measurements are feasible (see [1] for further details).

\section{1: Error Compensation}

Although proof-of-concept data obtained in 1995 was very encouraging, the accuracies obtained were far from those needed to be practical for machine automation purposes. They were, however, sufficient for other applications, such as personnel tracking in unstructured environments.

Typical solid-propagation measurement errors when penetrating a $500 \mathrm{~mm}$ thick slab of concrete were on the order of $800 \mathrm{~mm}$. That is, the measured distance was $800 \mathrm{~mm}$ longer than the true distance between the transmitter and receiver. This error was not affected by the range between the transmitter and receiver, thus confirming that most of the error was attributed to the slower phase velocity of the signal as it propagated through the concrete. Thus, a clear path was identified by which the phase propagation error could be compensated. Briefly the approach involves:

1) The development of empirically based statistical models of the electromagnetic characteristics for a large class of construction materials at varying frequencies of transmission and

2) A method by which a real-time mathematical model of the construction site (in the form of CAD solid model elements) can be updated with sufficient robustness to reflect the as-built condition of the site and the material specification of the various components.

Given this foundation it is possible to develop a software algorithm which takes into account the site geometry and material properties and predicts the delay in the arrival of the transmitted pulse due to propagation through engineering materials between the transmitter and receiver. In a more sophisticated variant of this approach all of the physical phenomena associated with wave propagation (including scattering, beating, reflection, and diffraction) may be taken into account and the result used to correct the initial measurement. With sufficient local processing power (in the form of a low power CMOS parallel processor array) such compensations could be made in a kinematic sense with update rates in excess of the 10 to $20 \mathrm{~Hz}$ commonly associated with real-time machine control.

Work in 1996 and 1997, which forms the basis of this paper, concentrated on defining propagation and error characteristics of spread spectrum signal penetration through construction materials as a function of the type of material (e.g. glass, concrete, wood etc.), frequency bandwidth, power, signal-to-noise ratio (and techniques, both physical and analytical for improving same), and obstacle geometry.

The resulting experimental data -- which encompass behavior across $8 \mathrm{GHz}$ of bandwidth and comprise one of the most complete sets of information concerning electromagnetic (EM) attenuation in construction materials -- form the basis for the development of autocompensation algorithms which will account for propagation delays as the signal passes through different materials. It is the propagation delay component that accounts for most of the range error. 
Ongoing research in 1997 is being directed to developing a range-error compensation model based on non-dispersive ray tracing techniques, which heretofore have largely been used for computer graphics rendering. In this work, CAD models of simulated construction sites are being developed and material characteristics, based on the extensive empirical EM material data acquired in 1996, are being attached to various entities in the CAD model. This model will be used to estimate range error in calculated position determined using the NLS system. It will thus allow conclusions to be drawn concerning the accuracy achievable through NLS, and its limitations and possibly will identify avenues for further resolution enhancement. The more complex phenomenon of EM wave propagation in dispersive media will be addressed in 1998.

\subsection{Description of the Invention}

The NLS measurement system is based on a variation of a an ultra-wideband synthetic aperture radar in which the receiving antenna was not collocated with the transmitting antenna, as is the case for most traditional bi-static radar systems.

In this study the receiving antenna comprised a roving unit that was located on the opposite side of the material target. In this sense, it was "cooperatively" working with the system, receiving the transmitted signal as opposed to the reflected signal. The microwave transmission system was based on a Hewlett-Packard HP8530 network analyzer/microwave receiver combined with an HP 83623A frequency synthesizer, HP 8511A frequency converter, and an HP 85330A multiple channel controller. A 486 PC-based computer network performed radar control functions, while data calibration and data management were handled with a Pentium PC.

The wideband pulse modulators used for hardware gating and the computer software for system control and data processing were custom developed by Flam and Russell, Inc. of Horsham, PA. MIT Lincoln Lab developed the laboratory test system (detailed in Figure 2.1) and cooperated with NIST researchers on all aspects of the laboratory research and subsequent data reduction. The radar was field-portable with the electronics and computational hardware based in a mid-size van. Table 2.1 lists characteristics of this system. The unit is fully polarimetric and operates over two frequency bands $(0.5-2 \mathrm{GHz}$ and $2-18 \mathrm{GHz})$.
In order to measure distance through an obstruction (e.g. a concrete wall), two separate antennas are used, as shown in Figures 2.1, 2.2, 2.3 and 2.5. The receiver becomes a "roving" unit whose position is to be determined. For the situation depicted in Figure 2.1 it is important to recognize that it is the time of flight, determined by performing a chirp- $Z$ transform on the in-phase and quadrature components of the transmitted and received frequency spectrum, that is being measured.

The measured time of flight then converts directly to a straight line distance between the transmitter and receiver as follows:

$$
\begin{aligned}
& x=c \cdot \delta t \\
& x=\text { straight line distance }(\mathrm{m}) \\
& \mathrm{c}=\text { speed of light }(300,000,000 \mathrm{~m} / \mathrm{s}) \\
& \delta \mathrm{t}=\text { time of flight }(\mathrm{s})
\end{aligned}
$$

Equation (2.1) can determine only the straight line distance from the transmitting antenna to the roving antenna. In order to acquire a unique three dimensional position at least three transmitters are required. The position can then be determined based on three dimensional triangulation. In such calculations it is assumed that precision (mm level) surveys will have been made to establish the benchmark positions of the transmitting antennas.

Returning now to the determination of time of flight, the following simplified summary will assist in helping to understand the NLS concept. First, since accuracy is of primary concern in the design of a precision surveying instrument we concentrate on this first. Speed (update rate) of position is also of great importance for those items requiring real-time feedback (e.g.

Table 2.1: RF Transmission System

\begin{tabular}{|l|l|}
\hline Frequencies & $0.5-2 \mathrm{GHz}, 2-18 \mathrm{GHz}$ \\
\hline Bandwidth & Antenna limited \\
\hline Waveform & Gated CW \\
\hline Pulse Width & $10 \mathrm{~ns}$ to $500 \mathrm{~ns}$ \\
\hline PRF & $50 \mathrm{kHz}$ to $5 \mathrm{MHz}$ \\
\hline Polarization & Fully polarimetric \\
\hline Output Power & $20 \mathrm{dBm}$ \\
\hline Dynamic Range & $80 \mathrm{~dB}$ \\
\hline Noise Floor & $-100 \mathrm{dBm}$ \\
\hline
\end{tabular}




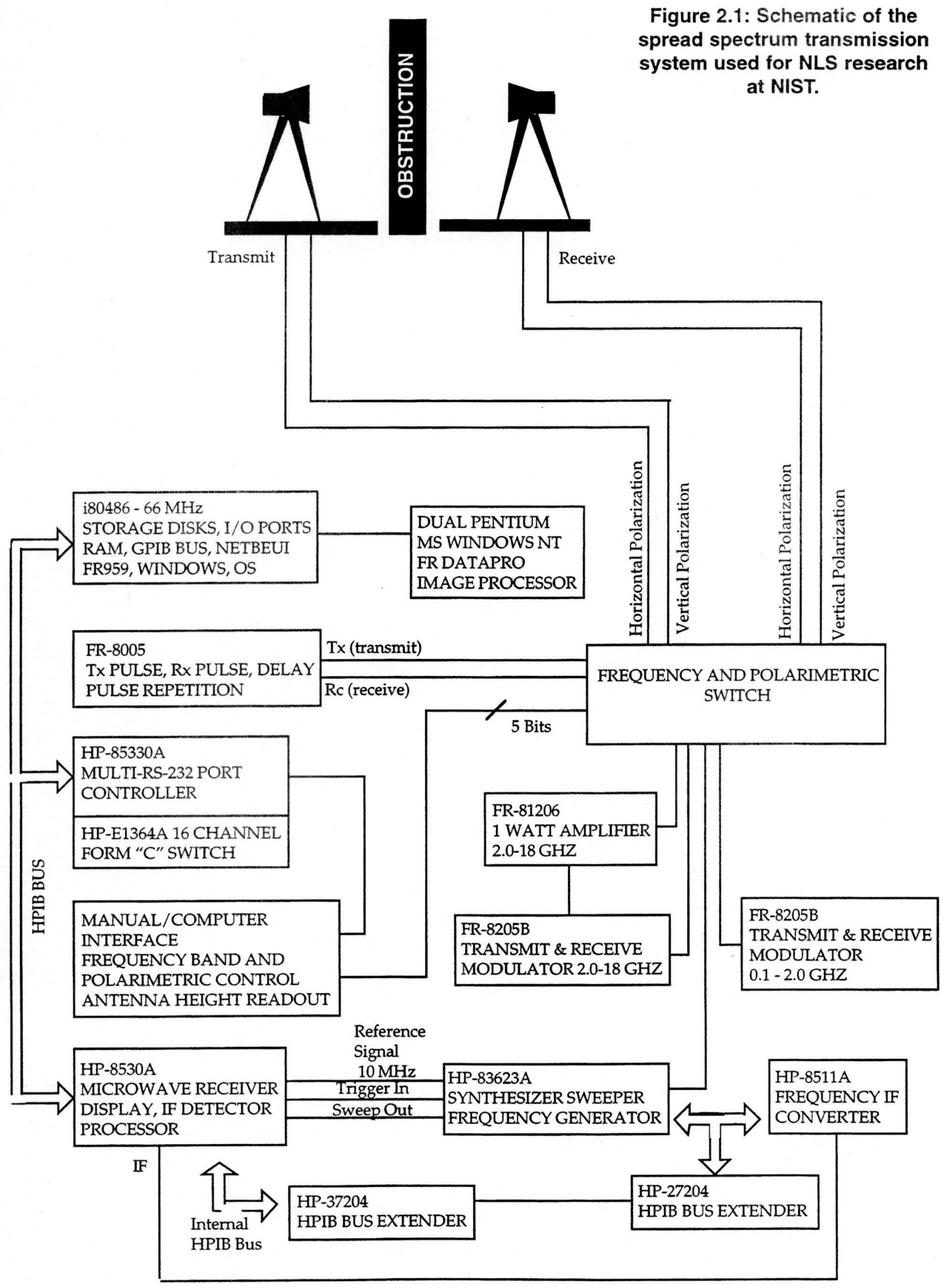




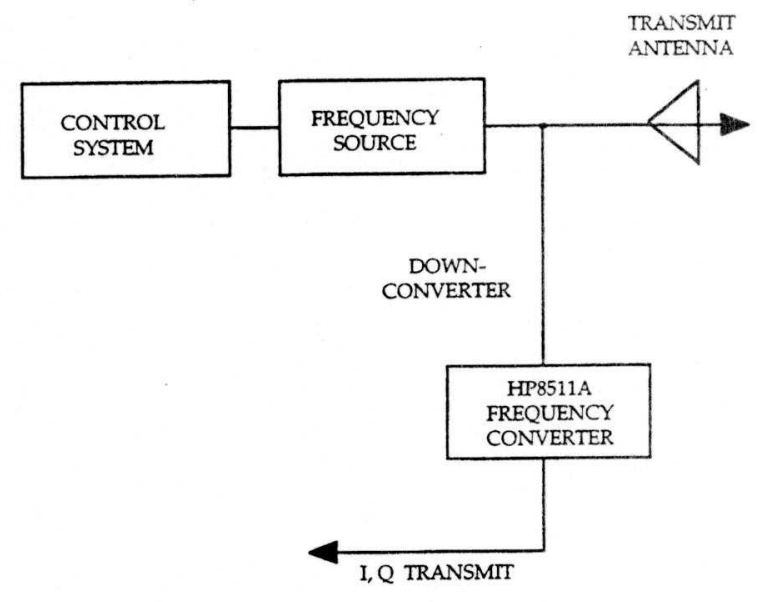

Figure 2.2: Simplified schematic of the transmission system and means for characterizing the transmitted signal.

automated operation of construction machinery). However, as will become evident, speed is primarily controlled by processor speed, which improves every year. Therefore, the approach discussed below, while designed primarily for accuracy, will nonetheless provide the algorithmic basis for real-time processing as local embedded microprocessors become faster.

Traditional imaging radars and scatterometers [4] make use of a swept frequency for the generation of a frequency response spectrum. An alternative, discrete approach [5] was used for the NIST NLS studies. In this approach, the response of the system is obtained at individual continuous wave frequencies. The discrete step size is user-definable, but for the NIST tests, 401 points were used for the 0.5 to $2.0 \mathrm{GHz}$ experiments and 801 points for the 3.0 to $8.0 \mathrm{GHz}$ experiments. This produced a frequency step size of approximately 3.74 $\mathrm{MHz}$ and $6.24 \mathrm{MHz}$, respectively. Thus, for the 0.5 to $2.0 \mathrm{GHz}$ case, the frequency response spectra was discretely assembled by means of the results of 401 independent $\mathrm{CW}$ single-frequency tests, each at a successively higher frequency.

Figure 2.2 is a schematic of the transmitter subsystem. In the actual system, the computer directs the HP$83623 \mathrm{~A}$ to generate the specified frequency which is then amplified and sent to the transmission antenna. This same signal is also tapped to an RF-to-IF converter (HP-8511A) where the IF signal is then sent to the network analyzer (HP-8530A) for for signal detection. The magnitude and phase of the transmitted signal are

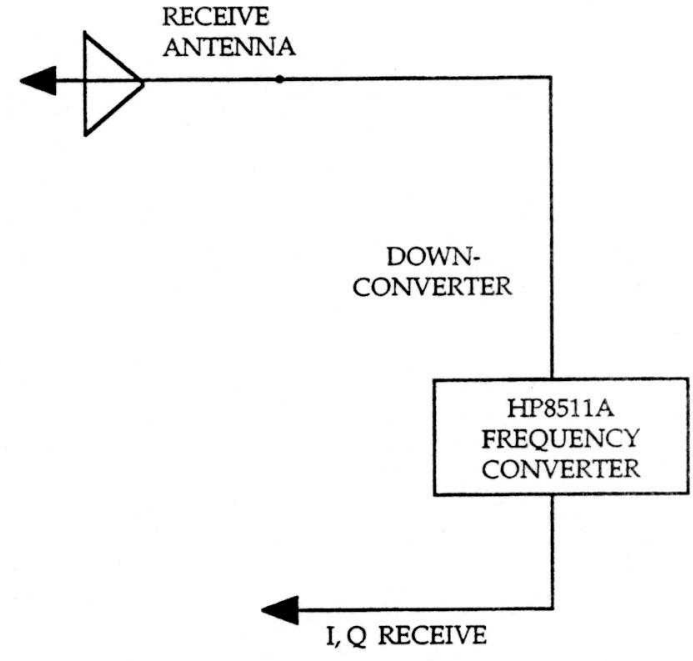

Figure 2.3: Simplified schematic of the receiver system and means for characterizing the received signal.

then extracted in a fashion following that depicted in Figure 2.4. This procedure is known as "quadrature" detection and can be thought of as a mixing operation that translates the received (tapped) signal to baseband to recover amplitude and phase in the form of quadrature components.

The in-phase component (or "I" component) is created by multiplying the transmitted signal by a reference signal generated by the mixer oscillator. The input signal is defined as:

$B_{1} \cos \left(2 \pi f t+\phi_{1}\right)$

The mixer reference signal, generated at the same frequency, but different amplitude and phase, is:

$B_{2} \cos \left(2 \pi f t+\phi_{2}\right)$

The result of multiplying the two signals is:

$B_{1} B_{2} \cos \left(2 \pi f t+\phi_{1}\right) \cos \left(2 \pi f t+\phi_{2}\right) \mathrm{Eq}(2.4)$ 


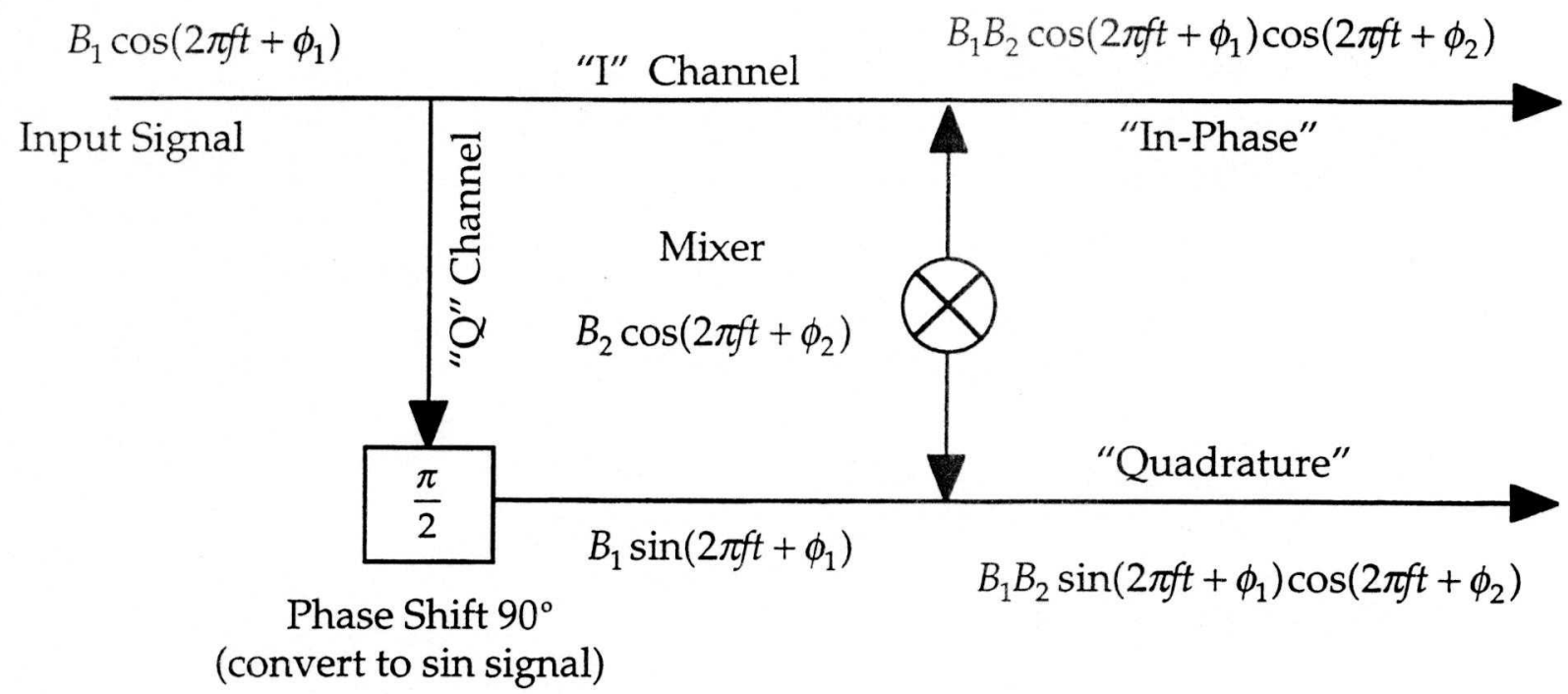

Figure 2.4: Schematic representation of a down conversion "mixer" used for quadrature detection in both the transmitted and received signals. In the NIST NLS tests, a common mixer oscillator was used as the reference for both signals.

Eq (2.3) can be expanded by means of standard trigonometric identities to yield:

$$
B_{1} B_{2}\left(\begin{array}{l}
\frac{1}{2} \cos \left(\omega t+\phi_{1}-\left(\omega t+\phi_{2}\right)\right) \\
+ \\
\frac{1}{2} \cos \left(\omega t+\phi_{1}+\left(\omega t+\phi_{2}\right)\right)
\end{array}\right)
$$

where term $\omega$, the cyclic frequency, is used interchangeably with the term $2 \pi \mathrm{f}$. By collecting terms this can be re-written as $\mathrm{Eq}(2.6)$ :

$$
B_{1} B_{2}\left(\frac{1}{2} \cos \left(\phi_{1}-\phi_{2}\right)+\frac{1}{2} \cos \left(2 \omega t+\phi_{1}+\phi_{2}\right)\right)
$$

The first component in $\mathrm{Eq}(2.6)$ represents the $\mathrm{DC}$ signal or "bias" component and does not depend on frequency. It does, however, contain information relating to the phase difference between the input (transmitted) and reference signals.
In order to extract on the first part, the composite signal is typically run through a low pass filter. The output from the low pass filter, for the in-phase component is:

$$
\frac{B_{1} B_{2}}{2} \cos \left(\phi_{1}-\phi_{2}\right) \equiv I
$$

where the "I" is used to indicate that this term characterizes the real or "in-phase" component of the input signal. In a similar fashion the quadrature (or imaginary) component of the complex input signal can be generated by phase shifting the input signal by $\pi / 2$ radians. The resulting "quadrature" signal is:

$$
B_{1} B_{2}\left(\frac{1}{2} \sin \left(\phi_{1}-\phi_{2}\right)+\frac{1}{2} \cos \left(2 \omega t+\phi_{1}+\phi_{2}\right)\right)
$$

Passing this signal through a low-pass filter yields the "quadrature" (or imaginary) component of the input signal as: 


$$
\frac{B_{1} B_{2}}{2} \sin \left(\phi_{1}-\phi_{2}\right) \equiv Q
$$

The transmitted signal, at the discrete frequency, $\mathrm{f}_{0}$, can now be completely characterized as:

$$
\mathrm{S}_{\mathrm{T}}=\mathrm{I}_{\mathrm{T}}+\mathrm{j} \mathrm{Q}_{\mathrm{T}}
$$

where

$$
j=\sqrt{-1}
$$

The transmit signal amplitude is:

$$
A_{T}=\sqrt{I_{T}^{2}+Q_{T}{ }^{2}}
$$

The same procedure (quadrature detection) is applied to the received signal, as depicted in Figure 2.3. In this situation there is an important additional consideration in that, although we are transmitting continuously at the selected discrete frequency, $\mathrm{f}_{0}$, there may (and and almost always will) be other components of the signal, at different frequencies and phases, resulting from multipath reflections, refraction and other dispersive phenomena. In order to eliminate these unwanted secondary signals, the receiver is tuned to listen at the specified frequency, $\mathrm{f}_{0}$, by means of a narrow band pass filter. The band error typical of the NIST tests was $\pm 20 \mathrm{KHz}$. In the particular implementation described in Figure 2.3, the same mixer reference signal is used for quadrature detection as that shown in Figure 2.2. The resulting received signalis given by:

$$
\mathrm{S}_{\mathrm{R}}=\mathrm{I}_{\mathrm{R}}+\mathrm{j} \mathrm{Q}_{\mathrm{R}}
$$

At this point it will be useful to switch to phasor notation to describe the process by which the attenuation and phase angle are derived. Phasor notation is based on the equality:

$$
e^{j \theta}=\cos \theta+j \sin \theta
$$

Using this notation, the received signal is:

$$
\begin{aligned}
& \tilde{B}=\operatorname{Bcos}\left(2 \pi f_{0}+\phi_{B}\right) \\
& =\operatorname{Real}\left(B e^{j\left(2 \pi f_{0}+\phi_{B}\right)}\right) \\
& =\operatorname{Real}\left(B^{j \phi_{B}} e^{j\left(2 \pi f_{0}\right)}\right)
\end{aligned}
$$

and the transmitted signal is:

$$
\begin{aligned}
& \tilde{A}=\operatorname{Acos}\left(2 \pi f_{0}+\phi_{A}\right) \\
& =\operatorname{Real}\left(A e^{j\left(2 \pi f_{0}+\phi_{A}\right)}\right) \\
& =\operatorname{Real}\left(A e^{j \phi_{A}} e^{j\left(2 \pi f_{0}\right)}\right)
\end{aligned}
$$

The ratio of the received to transmitted signals, as depicted in Figure 2.5, is:

$$
\tilde{C}_{R A W}=\frac{\tilde{B}_{\text {RECEIVE }}}{\tilde{A}_{\text {TRANSMIT }}}
$$

If we substitute $\mathrm{Eqs}(2.14$ and 2.15) into $\mathrm{Eq}(2.16)$ we obtain:

$$
\begin{aligned}
& \frac{\tilde{\mathrm{B}}}{\tilde{\mathrm{A}}}=\frac{\mathrm{B} \mathrm{e}^{\mathrm{j} \phi_{\mathrm{B}}}}{\mathrm{Ae}^{\mathrm{j} \phi_{\mathrm{A}}}} \\
& =\frac{\mathrm{B}}{\mathrm{A}} e^{j\left(\phi_{B}-\phi_{A}\right)}
\end{aligned}
$$

The amplitude ratio, $\mathrm{B} / \mathrm{A}$, in the above equation represents the attenuation of the signal as a result of propagation through a dispersive medium. The ratio varies from 0.0 to 1.0 with 1.0 representing transmission in a vacuum (ideal transmission) and 0.0 representing complete attenuation (absorption and reflection) by the media. The phase angle difference in the exponent is a measure of the time of flight between the transmitter and receiver, including dispersion-related group velocity effects as the signal propagates through engineering materials.

From Eqs(2.13 and 2.17): 
Transmit $\tilde{\text { A }}$ [Vector with Amplitude and Phase] at Single Frequency

2: Repeat at

Multiple

Frequencies to

Define $\mathrm{C}$ as a

Function of

Frequency

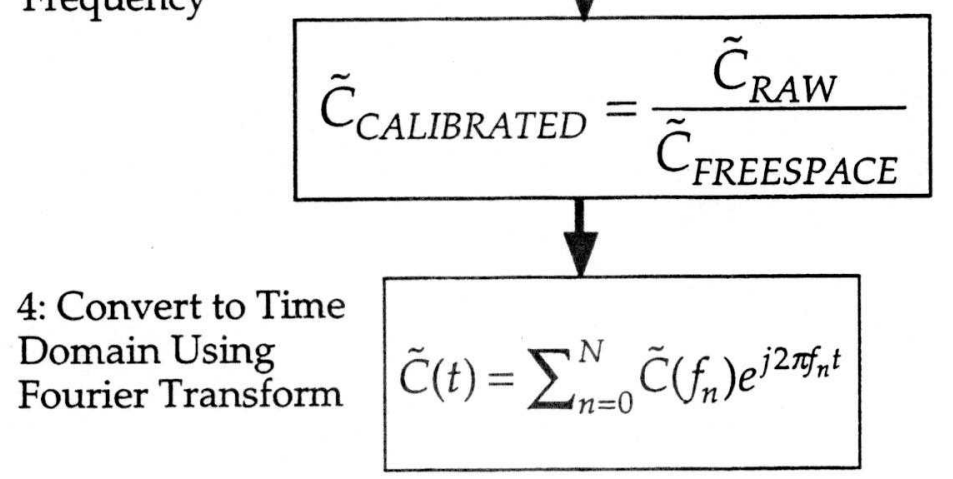

1: Ratio of Transmitted to Received Signals is a Measure of their Amplitude and Phase Difference
3: Use Free Space Measurement to Divide Out the Hardware System Response

Figure 2.5: Impulse synthesis approach used in the NIST NLS tests. Composite signals with bandwidths of 1.5 and $5 \mathrm{GHz}$, respectively, were achieved by recording the response for discrete single frequency transmissions. The composite time domain response was synthesized via chirp- $Z$ transform from the discrete frequency response spectrum.

$\frac{\tilde{\mathrm{B}}}{\tilde{\mathrm{A}}}=\frac{B \cos \phi_{B}+j B \sin \phi_{B}}{A \cos \phi_{A}+j A \sin \phi_{A}}$

$\mathrm{I}_{\mathrm{R}}=B \cos \phi_{B}$

$Q_{R}=j B \sin \phi_{B}$

$\mathrm{I}_{\mathrm{T}}=A \cos \phi_{A}$

$Q_{A}=j A \sin \phi_{A}$

From $\mathrm{Eq}(2.18)$ it is apparent that the values of $\mathrm{I}_{\mathrm{R}}, \mathrm{Q}_{\mathrm{R}}$, $\mathrm{I}_{\mathrm{T}}$, and $\mathrm{Q}_{\mathrm{T}}$ can be determined via quadrature detection

Eq (2.18) as expressed in Eqs(2.10) and (2.12) above. We can thus proceed directly to write that the ratio of received to transmitted signals is given by:

$$
\frac{S_{R}}{S_{T}}=\frac{\tilde{\mathrm{B}}}{\tilde{\mathrm{A}}}=\frac{I_{R}+j Q_{R}}{I_{T}+j Q_{T}}
$$

$\mathrm{Eq}(2.19)$ represents a complex division of the form [6]: 


$$
\begin{aligned}
& \frac{A}{C}=\frac{a+j b}{c+j d} \\
& =\frac{(a c+b d)+j(b c-a d)}{a^{2}+b^{2}} \\
& =\frac{(a c+b d)}{a^{2}+b^{2}}+\frac{j(b c-a d)}{a^{2}+b^{2}}
\end{aligned}
$$

The solution for the ratio of the received to transmitted signals, at the discrete frequency, $\mathrm{f}_{0}$, being investigated as:

$$
\begin{aligned}
& \tilde{C}_{R A W}=\frac{S_{R}}{S_{T}}=\frac{\tilde{B}}{\tilde{A}}= \\
& \frac{\left(I_{R} I_{T}+Q_{R} Q_{T}\right)}{I_{R}{ }^{2}+Q_{R}{ }^{2}}+j \frac{\left(Q_{R} I_{T}-I_{R} Q_{T}\right)}{I_{R}{ }^{2}+Q_{R}{ }^{2}} \\
& =I_{R A W}+j Q_{R A W}
\end{aligned}
$$

As shown in Figure 2.5, this procedure is carried out for each discrete frequency of interest with the ultimate objective of building a discrete frequency response spectrum for a given bandwidth of interest. However, the results presented in $\operatorname{Eq}(2.21)$ represent uncalibrated, or "raw" data. There are a number of factors which need to be taken into account.

The most important of the corrections involves deviations in the signal propagation velocity through the atmosphere. As previously discussed, the objective is to determine the time of flight of the signal from the transmitting antenna to the receiving antenna. The conversion to distance involves the velocity of electromagnetic radiation in the atmosphere, as given by $\mathrm{Eq}(2.1)$. Small errors in the determination of the propagation velocity can lead to large errors in the straight line dis-

\begin{tabular}{|c|c|}
\hline \multicolumn{2}{|l|}{ where } \\
\hline$N=$ & scaled-up refractivity \\
\hline$p=$ & total pressure in millibars \\
\hline$e=$ & $\begin{array}{l}\text { partial pressure of water vapor } \\
\text { in millibars }\end{array}$ \\
\hline $\mathrm{T}=$ & $\begin{array}{l}\text { absolute temperature in } \\
\text { degrees Kelvin }\end{array}$ \\
\hline$c_{\text {vacuum }}=$ & $\begin{array}{l}\text { speed of light in a } \\
\text { vacuum }\end{array}$ \\
\hline
\end{tabular}
tance estimate. Empirical correction factors have been derived for propagation of radio waves through the atmosphere as follows:

$$
\begin{aligned}
& N=\frac{77.6 p}{T}+\frac{373000 e}{T^{2}} \\
& n=1+\frac{N}{10^{6}} \\
& c_{\text {atmosphere }}=\frac{c_{\text {vacuum }}}{n}
\end{aligned}
$$

In addition there are also errors which result from the internal electrical nature of the radar instrument. For example, the length and efficiency of the coaxial cables which connect the antennas to the system and the efficiency with which signals are propagated through the various electrical subsystems.

Fortunately, there is an elegant, and simple method for accounting for all of these sources of error, both due to propagation through the atmospheric and from instrumental error, by means of a calibration procedure [5]. This involves the generation of a point for point discrete frequency response spectrum, using the same techniques outlined above, but with the important difference that the setup be a line of sight test over a known, precision surveyed bench. The bench consists of two benchmarks whose positions have been established relative to one another using standard total station surveying equipment. The resulting "freespace" response is used to calibrate the raw data by dividing the raw signal, at each discrete frequency, by the freespace result, as shown in Figure 2.5, following Equation(2.23):

$$
\tilde{C}_{\text {CALIBRATED }}=\frac{\tilde{C}_{\text {RAW }}}{\tilde{C}_{\text {FREESPACE }}}
$$


The freespace signal is defined as

$$
S_{F}=\tilde{C}_{F R E E S P A C E}=I_{F}+j Q_{F} \quad \text { Eq (2.24) }
$$

where the notation is the same as previously used to describe the normalized in-phase and quadrature components for the raw signal defined in Eq (2.21). The $\mathrm{F}$ subscripts in $\mathrm{Eq}(2.24)$ denote the freespace calibration. Equation 2.23 can now be rewritten as:

Eq (2.25)

$$
\begin{aligned}
& \tilde{C}_{C A L I B R A T E D}=\frac{\tilde{C}_{R A W}}{\tilde{C}_{F R E E S P A C E}}= \\
& \frac{I_{R A W}+j Q_{R A W}}{I_{\text {FREESPACE }}+j Q_{F R E E S P A C E}}= \\
& \frac{\left(I_{R A W} I_{F R E E}+Q_{R A W} Q_{\text {FREE }}\right)}{I_{\text {RAW }}{ }^{2}+Q_{\text {RAW }}{ }^{2}}+ \\
& j \frac{\left(Q_{\text {RAW }} I_{\text {FREE }}-I_{\text {RAW }} Q_{\text {FREE }}\right)}{I_{\text {RAW }}{ }^{2}+Q_{\text {RAW }}{ }^{2}} \\
& =I_{\text {CALIBRATED }}+j Q_{\text {CALIBRATED }}
\end{aligned}
$$

where

$$
\begin{aligned}
& I_{R A W}=\left(\frac{\left(I_{R} I_{T}+Q_{R} Q_{T}\right)}{I_{R}{ }^{2}+Q_{R}{ }^{2}}\right)_{R A W} \\
& Q_{R A W}=\left(\frac{\left(Q_{R} I_{T}-I_{R} Q_{T}\right)}{I_{R}^{2}+Q_{R}{ }^{2}}\right)_{R A W} \\
& I_{F R E E}=\left(\frac{\left(I_{R} I_{T}+Q_{R} Q_{T}\right)}{I_{R}{ }^{2}+Q_{R}{ }^{2}}\right)_{F R E E} \\
& Q_{F R E E}=\left(\frac{\left(Q_{R} I_{T}-I_{R} Q_{T}\right)}{I_{R}{ }^{2}+Q_{R}{ }^{2}}\right)_{F R E E}
\end{aligned}
$$

For the NLS tests conducted at NIST, the above calculations were performed in a post-processing mode since the data acquisition system was only designed to write one file at a time. Thus, the freespace response was always acquired prior to the commencement of any day's testing. Furthermore, a second freespace measurement was made at the end of each day to compare against any changes that might have occurred during the day. Subsequent to the freespace measurement, raw experimental data files were created for each material test following $\mathrm{Eq}(2.21)$. The final calibration, following $\mathrm{Eq}(2.25)$, was performed as part of an automated MatLab post-processing script that operated on all data files.

The calibrated frequency response spectra were stored as a $5 \mathrm{x} n$ matrix, where $\mathrm{n}$ was the number of discrete frequencies sampled. As previously described, 401 evenly spaced points were acquired for the 0.5 to. 0 $\mathrm{GHz}$ tests and 801 points were acquired for the 3.0 to $8.0 \mathrm{GHz}$ tests. For each frequency, the $\mathrm{I}$ and $\mathrm{Q}$ values ( $\mathrm{I}_{\text {CALIBRATED }}$ and $\mathrm{Q}_{\text {CALIBRATED }}$ (Eq.2.25) were stored in the second and third columns respectively. The fourth column contained the derived calibrated, normalized amplitude, as given by $\mathrm{Eq}(2.11)$. Specifically, for the calibrated signal, the amplitude is calculated as:

$$
\begin{aligned}
& A_{\text {CALIBRATED }}= \\
& \sqrt{\mathrm{I}_{\text {CALIBRATED }}{ }^{2}+Q_{\text {CALIBRATED }}{ }^{2}}
\end{aligned}
$$

This is a decimal value between 0.0 and 1.0 , with 1.0 meaning that there was no attenuation observed during the test for which a material specimen was placed between the transmitter and receiver, relative to the same freespace test. The decimal amplitude may easily be converted to decibels $(\mathrm{dB})$ :

$$
A(d B)=20 * \log _{10} A(\text { decimal })
$$

In addition, the phase angle (radians) for the calibrated response signal was stored in the fifth column in each calibrated data file. The phase angle is:

$$
\phi_{\text {CALIBRATED }}=\tan ^{-1}\left(\frac{Q_{\text {CALIBRATED }}}{I_{\text {CALIBRATED }}}\right)
$$


In the attenuation versus frequency plot presented later in the paper the frequency (in $\mathrm{GHz}$ ) is plotted on the $\mathrm{x}$-axis while the decimal version of $\mathrm{Eq}(2.26)$ is plotted on the y-axis. Efforts have been made to fit simplified closed form solutions to these curves for the purposes of characterizing the attenuation characteristics of a broad range of materials over a very wide frequency bandwidth while maintaining a manageable and compact means for accessing that data (see Table 7.1). Such equations are available, e.g., for all of the materials listed in Table 5.2 [3].

\section{3: Antenna Descriptions}

Two antenna geometries have been used for the NIST test program. The first, originally manufactured by Watkins-Johnson* and now fabricated by Condor Systems, had a bandwidth of $1.5 \mathrm{GHz}$ between 0.5 and $2.0 \mathrm{GHz}$. The second, manufactured by Flam and Russell, had a bandwidth of $5 \mathrm{GHz}$ from 3.0 to $8.0 \mathrm{GHz}$. Where polarization capability existed, the V-V polarization configuration was employed for the NIST tests, as initial studies [1] clearly indicated that multipath distortion due to ground bounce was minimized through the use of a vertically polarized plane wave transmission.

These antennas provide high-gain directional patterns over multi-octave bandwidths. Each pyramidal horn (Figure 3.1) has two orthogonally placed input feeds which provide the capability for simultaneous horizontal and vertical polarization. For the attenuation tests reported herein, only the vertical polarization was used. A typical implementation is shown in Figures 3.2 and 3.3 , where attenuation and distance measurements are being taken for a composite specimen consisting of

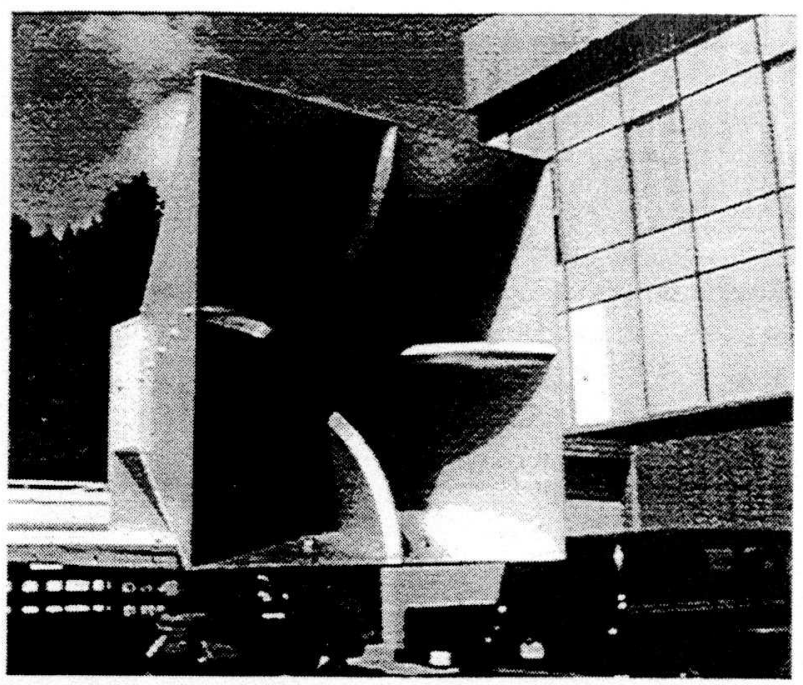

Figure 3.1: Quadridged 0.5 to $2.0 \mathrm{GHz}$. Vertical polarization, used for NIST tests, used only the two central vertical ridges.

three back-to-back masonry block walls.

A relatively broad area of illumination is provided by this design. Although signal power is reduced by approximately one half $(3 \mathrm{~dB})$ by an azimuth angle of 30 degrees to either side of center, considerable signal strength can still be measured at azimuth angles of 90degrees. Because of the relatively different radiation pattern geometry for this antenna, as contrasted with that for the 3.0 to $8.0 \mathrm{GHz}$ diagonal horn described below, results pertaining to attenuation of transmitted power, tend to be antenna specific.

For tests in the range of 3.0 to $8.0 \mathrm{GHz}$ FR6400 class range illumination horns from Flam \& Russell,

Figure 3.2 (Right):

Experimental calibration of a composite wall consisting of three back-to-back 76 $\mathrm{mm}$ brick walls. The wall sections measure 1 meter square. The 3 $\mathrm{mm}$ thick aluminum panel in background served as a multipath signal shield.

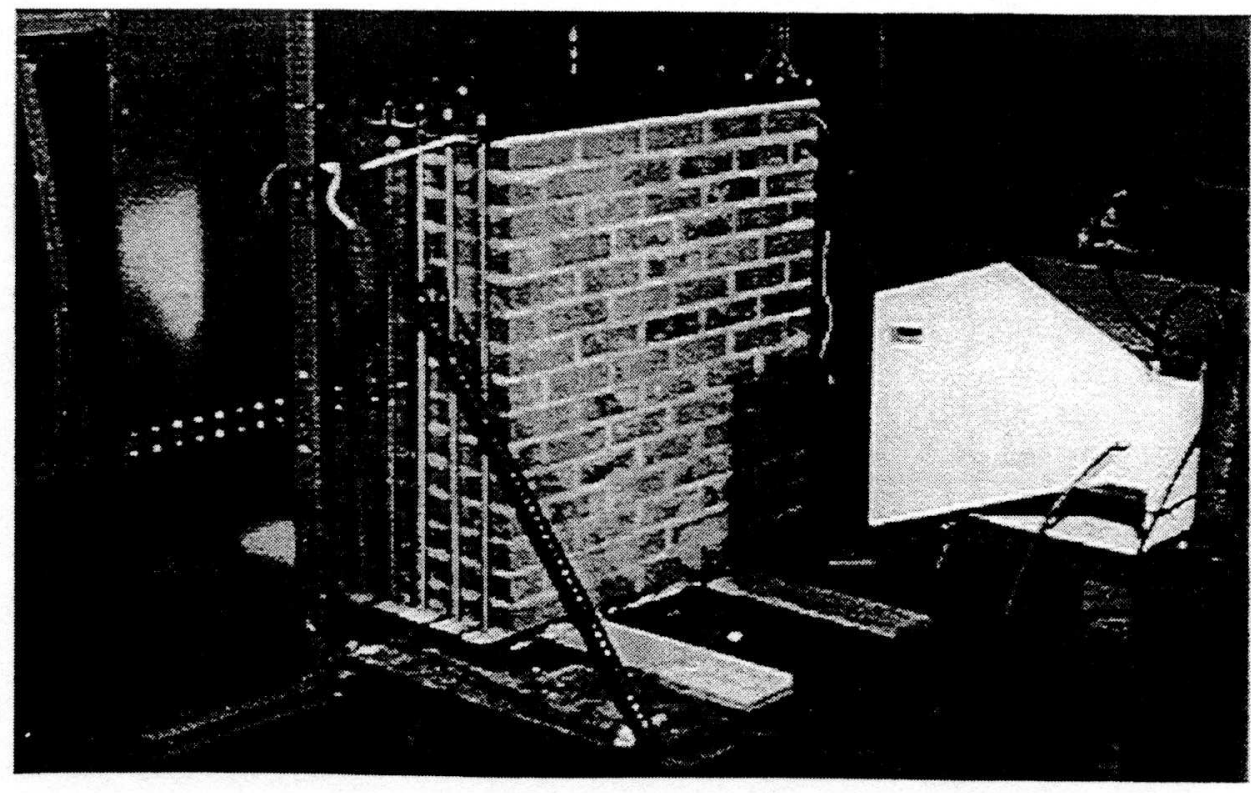




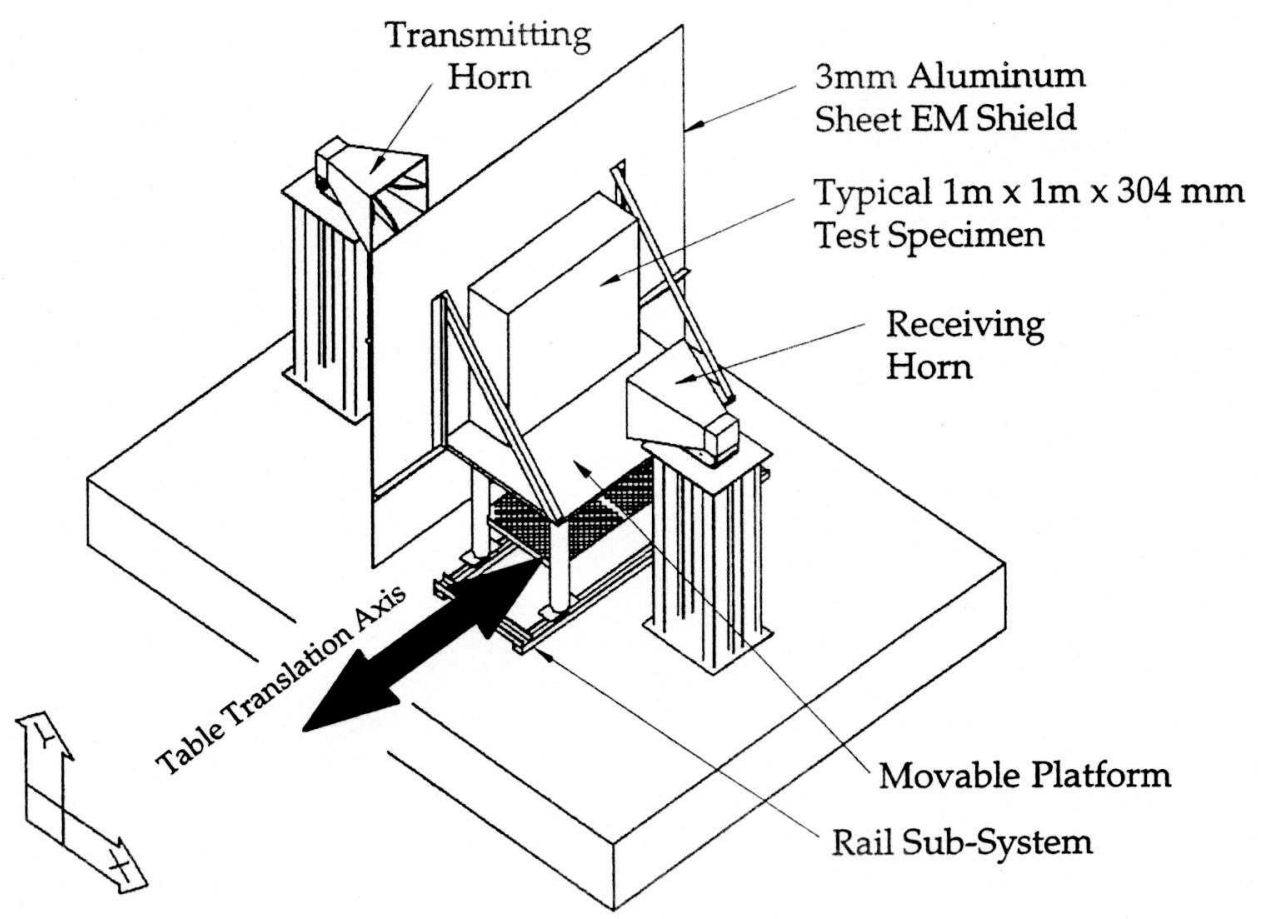

Figure 3.3: Isometric view of test stand for Phase 2 NLS tests. Mobile table is shown with specimen in place on opposite side of EM shield from transmitting horn.

Inc. were used. These horn antennas produce radiation patterns with low sidelobe structure, nearly circularly symmetric beam, and nominally constant gain and beamwidth.

The particular model used for the NLS tests was the FR-6415 3.0 to $8.0 \mathrm{GHz}$ horn, which was designed to serve as a source antenna in the measurement of antenna radiation patterns or as transmit and receive antennas in the measurement of radar cross-section data. These antennas exhibit significantly reduced Eplane sidelobes typically associated with horn antennas. The FR-6415 incorporates aperture defocusing to produce minimal variations in gain and beamwidth over a frequency range of 2.5:1.

Figure 3.4 shows a typical laboratory setup involving the FR-6415 in which the effect of a $203 \mathrm{~mm}$ thick reinforced concrete wall on time-of-flight distance measurement is being conducted. A frequency response spectrum was generated from 3.0 to $8.0 \mathrm{GHz}$ with this system.

\section{4: Test Fixtures}

Because of the large number of test specimens, and the representative full-size thicknesses used for the various materials, a test set-up was designed that made use of an indoor laboratory at NIST equipped with an overhead crane. The set-up is shown in isometric view in Figures 3.3 and 3.4 .

Previous tests with the radar system [1] had shown the problematic nature of multipath signals reaching, and distorting, the straight line (through the specimen) transmission signal. Calculations showed that an electromagnetic shield, in the form of a roughly $2.5 \mathrm{~m}$ square, $3 \mathrm{~mm}$ thick aluminum panel would effectively separate any multipath signals from the data except for those due to internal reflections within the specimen thickness. This was accurate provided the transmitting and receiving horns were relatively close to the test article. The distance selected was $1 \mathrm{~m}$ from the backplane of the EM shield to the aperture of each antenna. This meant that the distance from the receiving horn to the back side of the specimen varied, depending on the specimen thickness.

The EM shield contained a $914 \mathrm{~mm}$ square "window" on centerline through which the transmitted signals could pass unobstructed, as shown in Figures 3.3 and 3.4. Multipath signals diffracting around the edges of the shield arrived significantly later (in the time domain) and were thus easily eliminated using gating windows. 


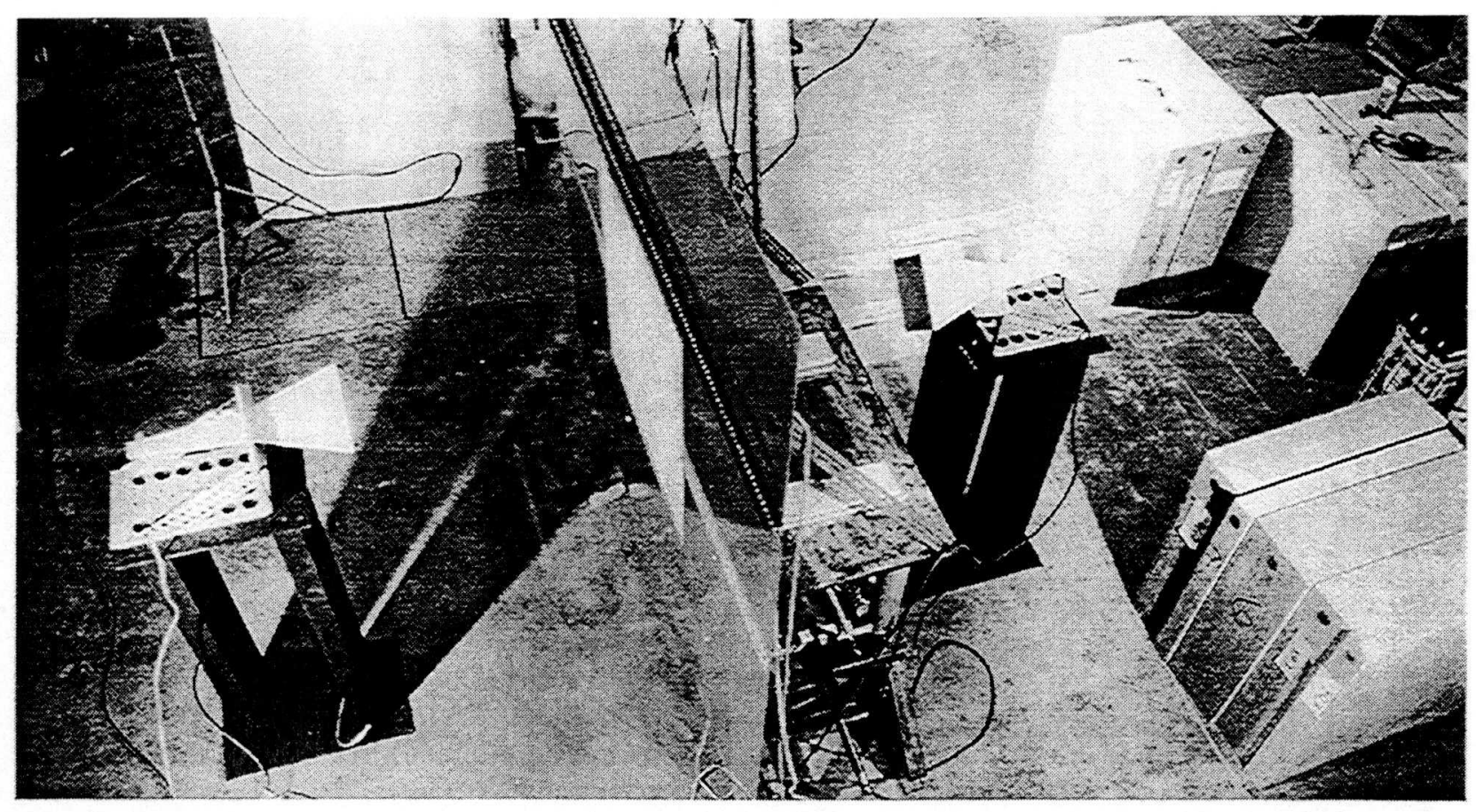

Figure 3.4: Oblique photo of the indoor NLS test range at NIST. The transmitting antenna is on the left. The receiving antenna (at right) was positioned behind an aluminum shield to insure that the data were not corrupted by multipath signals. The entire center section moved laterally, so that effects of material variance (inhomogeneity) could be investigated.

In order to gain statistical data concerning the variability of the measured distance with respect to the spatial and compositional variability of the various materials, the entire specimen carrier platform was mounted to a rail system that permitted translation of the specimen perpendicular to the line of signal transmission. In this manner, several duplicate sets of tests could be conducted for a single specimen, but with different sections of the specimen being located on the centerline of the transmission path.

\section{5: Specimen Design}

A total of 9 different materials were tested in this series. A summary of these is presented in Table 5.1. More detailed statistics are given in [3]. The test articles included common construction materials such as brick, masonry block, plain concrete, reinforced concrete, glass, lumber, plywood, drywall, and reinforcing rods. At least three different thicknesses, and in some cases four, were tested for each of the above materials. In addition, the lumber and plywood specimens were tested in both a wet and dry state.

Of particular interest is the list of plain concrete specimens in Table 5.1. Because of the widely varying nature of this common composite, efforts were made to construct an experiment matrix that captured some of the most important variables. These included aggregate size, water/cement ratio, and slump. The full material design matrix included high and low values for each of the above three parameters plus three panel thicknesses for a total of 24 plain concrete specimens. Reinforced concrete and reinforcing rod grids (in air) were investigated separately in order to quantify the affects of the reinforcing.

All specimens had a $1 \mathrm{~m}$ by $1 \mathrm{~m}$ cross section. Design thicknesses varied from as little as $6 \mathrm{~mm}$ to as much as $600 \mathrm{~mm}$ depending on the material and typical usage values at commercial and residential construction sites. Complete details on specimen geometry, material properties, as well as calibrated attenuation data from 0.5 through $8 \mathrm{GHz}$ are given in [3].

\section{6: Test Protocol}

The data for each test consisted of a calibrated frequency response spectrum. The theory and hardware instrumentation which allows for the generation of this spectra were presented in Section 2. These techniques were implemented in an automatic fashion via computer control. 
Table 5.1: Design Material Properties for Phase 2 NLS Tests

BFRL/Construction Automation Program

NLS Phase II Test Specimen Matrix

* All specimens to be $1 \mathrm{~m} \times 1 \mathrm{~m}$ square.

* Receiver Unit to be electromagnetically isolated from multipath by means of an aluminum plate shield ( $3 \mathrm{~mm}$ thick sheet, extending $1 \mathrm{~m}$ minimum about a central $95 \mathrm{~cm} \times 95 \mathrm{~cm}$ square hole

* Transmission and receiver antennas located $1 \mathrm{~m}$ from opposite sides of the panels ( $2.0 \mathrm{~m}$ spacing between antennas)

* All tests are conducted using both frequency bandwidths available: 0.500 $2.0 \mathrm{Ghz}$ (set 1); 3-8 Ghz (set 2).

* For concrete specimens the following properties apply:

\begin{tabular}{|c|c|c|c|c|c|c|c|c|}
\hline Properties & Batch 1 & Batch 2 & Batch 3 & Batch 4 & Batch 5 & Batch 6 & Batch 7 & Batch 8 \\
\hline MSA & 0.5 & 0.5 & 0.5 & 0.5 & 1 & 1 & 1 & 1 \\
\hline w/c & 0.4 & 0.4 & 0.6 & 0.6 & 0.4 & 0.4 & 0.6 & 0.6 \\
\hline Slump & low & high & low & high & low & high & low & high \\
\hline \% Cement & 15.8 & 18.2 & 10.5 & 12.1 & 14.1 & 16.0 & 9.4 & 10.7 \\
\hline \% Water & 19.9 & 22.9 & 19.9 & 22.9 & 17.8 & 20.2 & 17.8 & 20.2 \\
\hline \% CA & 29.9 & 29.9 & 29.9 & 29.9 & 37.9 & 37.9 & 37.9 & 37.9 \\
\hline \% FA & 31.9 & 26.5 & 37.2 & 32.6 & 28.6 & 24.3 & 33.3 & 29.7 \\
\hline \% Paste & 35.7 & 41.1 & 30.4 & 35.0 & 31.9 & 36.2 & 27.2 & 30.9 \\
\hline
\end{tabular}

Batch weights, SSD Basis, kilograms per cubic meter $\left(\mathrm{kg} / \mathrm{m}^{\wedge} 3\right)$

\begin{tabular}{|c|r|r|r|r|r|r|r|r|}
\hline Cement & 497.6 & 572.5 & 331.7 & 381.7 & 445.3 & 504.7 & 296.7 & 336.5 \\
\hline Water & 199.2 & 228.9 & 199.2 & 228.9 & 177.8 & 202.1 & 177.8 & 202.1 \\
\hline CA & 813.3 & 813.3 & 813.3 & 813.3 & 1032.1 & 1032.1 & 1032.1 & 1032.1 \\
\hline FA & 835.9 & 695.0 & 973.8 & 853.7 & 750.3 & 637.3 & 873.9 & 777.6 \\
\hline Density & 86.9 & 85.5 & 85.8 & 84.4 & 89.1 & 88.0 & 88.2 & 87.0 \\
\hline
\end{tabular}

Coarse Aggregate: ASTM \#7 (Batch 1-4), ASTM \#57 (Batch 5-8)

For each bath there will be 3 thickness: $102 \mathrm{~mm}, 203 \mathrm{~mm}$, and $305 \mathrm{~mm}$

For the Remaining Engineering Materials the following characteristics apply:

\begin{tabular}{|l|r|r|r|l|}
\hline \multicolumn{1}{|c|}{ Material } & Thickness & Thickness & Thickness & Thickness \\
\hline $\begin{array}{l}\text { Reinf. Concrete } \\
\text { \# 1, Type A Concrete } \\
\text { 1\% Mesh, Grade 60 }\end{array}$ & 203.2 & N/A & N/A & N/A \\
\hline $\begin{array}{l}\text { Reinf. Concrete } \\
\text { \# 2, Type A Concrete } \\
\text { 2\% Mesh, Grade 60 }\end{array}$ & 203.2 & N/A & N/A & N/A \\
\hline Glass(Plain) & 6.4 & 12.7 & 19.1 & \\
\hline Drywall & 6.4 & 9.5 & 12.7 & \\
\hline Plywood & 6.4 & 12.7 & 19.1 & 31.8 \\
\hline Masonry Block & 203.2 & 406.4 & 609.6 & \\
\hline Southern Pine & 38.1 & 76.2 & 114.3 & 152.4 \\
\hline Brick & 88.9 & 177.8 & 266.7 & \\
\hline
\end{tabular}

The protocol for testing any specimen, however, consisted of the following steps:

Step 1: Acquire freespace response spectra. At the start and finish of each day of testing a response spectrum was acquired using the test setup configured as shown in Figure 3.4, but with no specimen loaded. This was later used in the calibration of the raw signal as previously discussed.
Step 2: Acquire response spectrum for the test specimen. This step follows the previously described theoretical protocol for discrete construction of the response spectrum but with one important variation. Instead of a single measurement being made at each frequency, an average of 128 samples of the response at each frequency was recorded. This was performed automatically and was done to increase the accuracy of each individual frequency response measurement. The same procedure was used for the freespace measurements. 


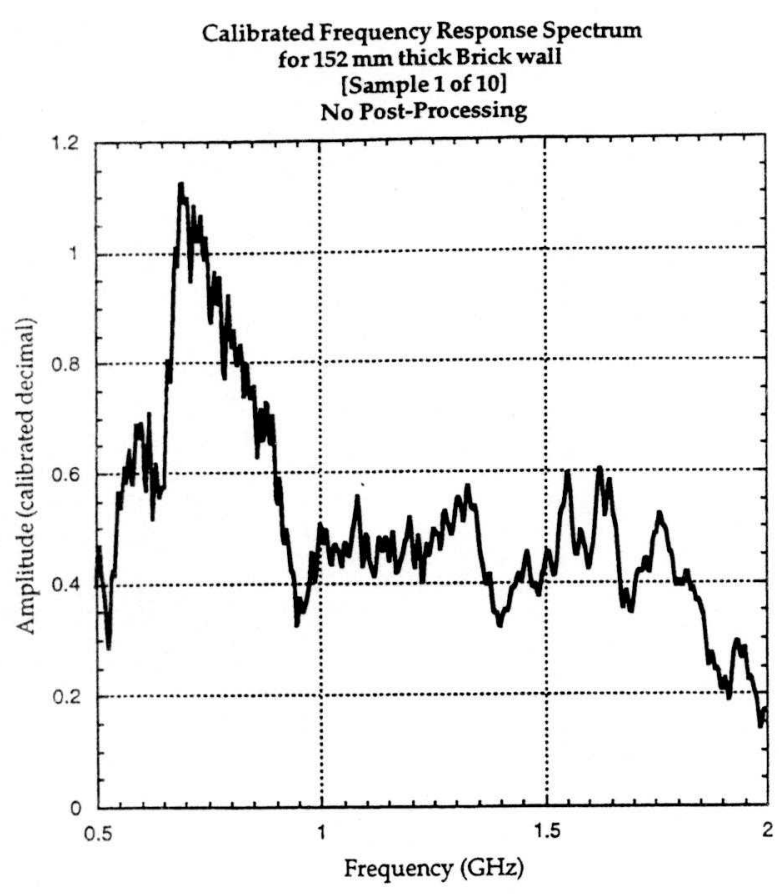

Figure 7.1: Calibrated frequency response spectrum for $152 \mathrm{~mm}$ thick brick wall (no post processing) for the 0.5 to $2.0 \mathrm{GHz}$ test series, Specimen \#1 of 10.

Step 3: Spatial variation tests. Ten separate tests were performed for each physical specimen. After each test the specimen platform was moved in $6 \mathrm{~mm}$ increments perpendicular to the signal transmission line between the two horns and the test repeated until a maximum displacement of $60 \mathrm{~mm}$ had been achieved. Each individual test was calibrated during the post processing cycle as described in Section 2.1. Using these ten separate tests, mean and standard deviation of the frequency response spectra for a particular specimen could be determined. It was this averaged frequency response spectrum that was used for subsequent time domain analyses.

\section{7: Post-Processing Procedures}

Several post-processing techniques were used to improve the utility of the attenuation spectra. First, the averaged frequency spectra for a given test specimen was converted to time domain using a chirp- $Z$ transform [7]. The implementation used for the tests reported here was an embedded function in DATAPRO (a proprietary code developed by Flam \& Russell) which was part of the experimental instrumentation. This transform can also be achieved using stand-alone post-processors such as, Matlab [8] and other digital signal processing packages. The chirp- $Z$ transform was used because it is

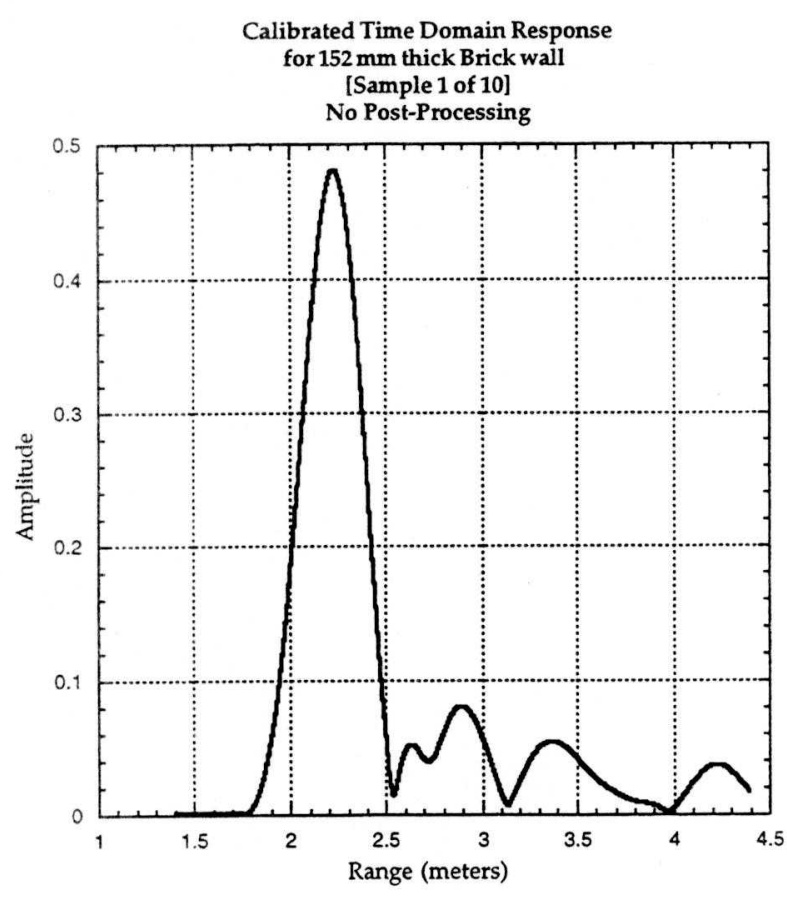

Figure 7.2: Calibrated time domain response for $152 \mathrm{~mm}$ thick brick wall (no post processing) for the 0.5 to $2.0 \mathrm{GHz}$ test series, Specimen \#1 of 10.

much faster than an FFT when prime length sequences are involved. In the case of the data presented here, there were 401 discretely sampled points which composed the 0.5 to $2.0 \mathrm{GHz}$ data and 801 points which composed the 3.0 to $8.0 \mathrm{GHz}$ data. A typical input file would be a frequency response spectrum like that shown in Figure 7.1 for the 0.5 to $2.0 \mathrm{GHz}$ test series. In this case, the data shown are for a $152 \mathrm{~mm}$ thick, double "wythe" brick wall, that is, a brick wall two blocks deep, similar to that shown in Figure 3.3. Using a chirp$z$ transform, the data in Figure 7.1 can be converted to the time domain plot shown in Figure 7.2. This is a particularly good data set, with very little attenuation involved. In fact, as can be seen for certain frequencies around $0.75 \mathrm{GHz}$ in Figure 7.1, the calibrated frequency response is actually slightly greater than 1.0 , implying that constructive "ringing" or "beating" is taking place within the wall. This will not normally be the case, especially with thicker, denser walls such as concrete.

The time domain plot shown in Figure 7.2 shows one predominant peak at a range of slightly more than two meters. Two observations need to be made at this point. First, a "time domain" data set implies amplitude as a function of time, usually expressed in seconds. In this case, the scale is actually in nanoseconds, where 1 


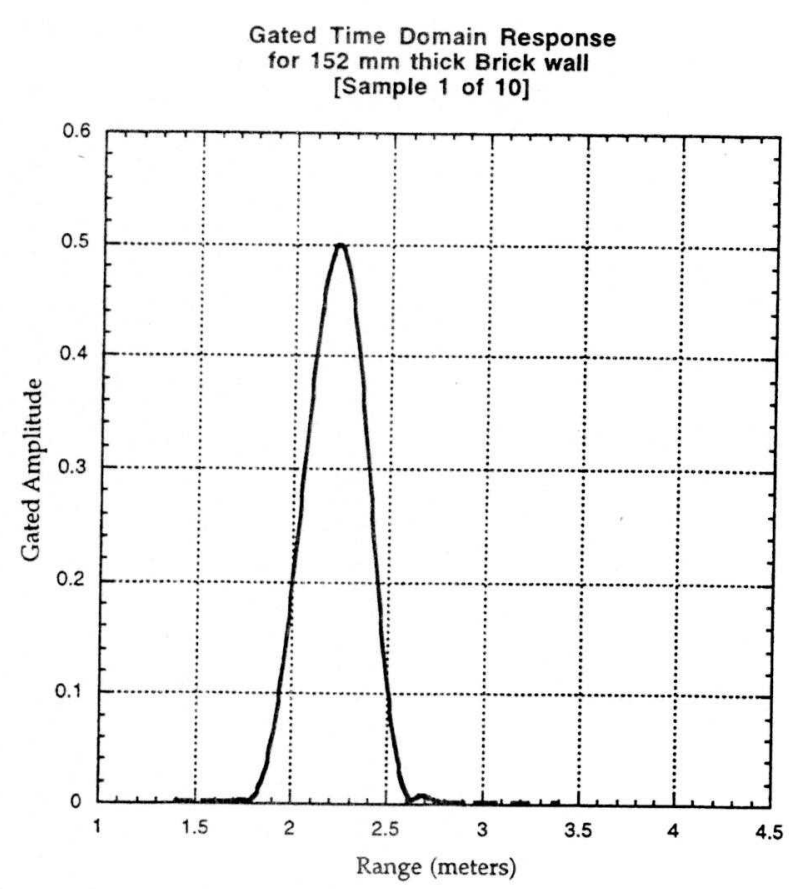

Figure 7.3: Gated time domain response for $152 \mathrm{~mm}$ thick brick wall for the 0.5 to $2.0 \mathrm{GHz}$ test series, Specimen \#1 of 10.

nanosecond of electromagnetic wave travel in free space is equivalent to approximately $305 \mathrm{~mm}$ (or $1 \mathrm{ft}$ ). This equivalence permits a recasting of the $\mathrm{x}$-axis scale in the more useful units of distance. The second point is that the true distance between the two antennas was pre-set at 2.000 meters. The peak in Figure 7.2 clearly is not at 2.000 meters. The difference, approximately $205 \mathrm{~mm}$, is almost entirely due to group-velocity lag time as the signal propagates through the target. During its transit through the brick wall, the signal travels (relative to our reference frame) at a velocity much smaller than the speed of light in a vacuum. For many engineering materials, for example reinforced concrete, the propagation velocity through the material can be as much as nine times less than the speed of light in a vacuum. This is important for the development of compensation algorithms to account for these errors in surveying instruments.

Adjacent to the predominant peak in Figure 7.2 are several ripples or side lobes. In general these will be much more pronounced in materials with higher water content and the signal to noise ratio is lower. It should also be noted that even for this case there is a substantial amount of noise in the frequency response spectra shown in Figure 7.1. This noise in the frequency spectrum is produced by both ringing (internal reflections within the target) and multipath signals. Since the true distance was known with high accuracy for these experiments, a range gate [9] on the time domain can be used to clean up the frequency response spectra.

The HP 8530A used for these experiments contains a gating feature which allows for selective viewing of individual portions of the time domain response. The "gate" is actually a band-pass shaped time domain filter. The user has direct control over the gate width and location including the selection of the gate start and stop times and the gate center. For the NIST data, the gate center was assigned to the maximum amplitude peak in the time domain response. The specific gating used was a 1 nanosecond wide Kaiser-Bessel window centered on the peak, which effectively considered only those data within a $6 \mathrm{~dB}$ amplitude variation from the peak. The result of the application of this gate in the time domain is shown in Figure 7.3 for the case of the brick wall previously considered.

An inverse transform of the time domain data yields the gated frequency response spectra (see Figure 7.4). This is a much cleaner representation of the attenuation characteristics of the transmitted signal with respect to frequency. The actual processing of the data proceeded in a step-wise fashion as follows:

- Define the time gates to use for each material sample using DATAPRO.

- Apply the time gating to each measurement with DATAPRO, and save the gated frequency and time domain data in Network Common Data Form (netCDF).

- Read the netCDF data and perform statistical analysis on the gated frequency and time domain data using MatLab.

- Write out the averages and standard deviations of the measurements for each sample in both netCDF and ASCII files.

The data presented in Figure 7.4 are typical of those obtained for all but the thickest concrete specimens tested. These data show, beyond doubt, that extremely small baseband transmission power levels ( 1 milliwatt was the level used in these experiments) are sufficient to penetrate nearly all common construction materials, other than solid metal. Reinforced concrete walls as thick as $500 \mathrm{~mm}$ have been successfully penetrated during the course of the NIST tests. For common residential construction, involving $2 \times 4$ frames, drywall, and either plywood, masonry block or brick exteriors, the materials are nearly "transparent," as far as NLS is 


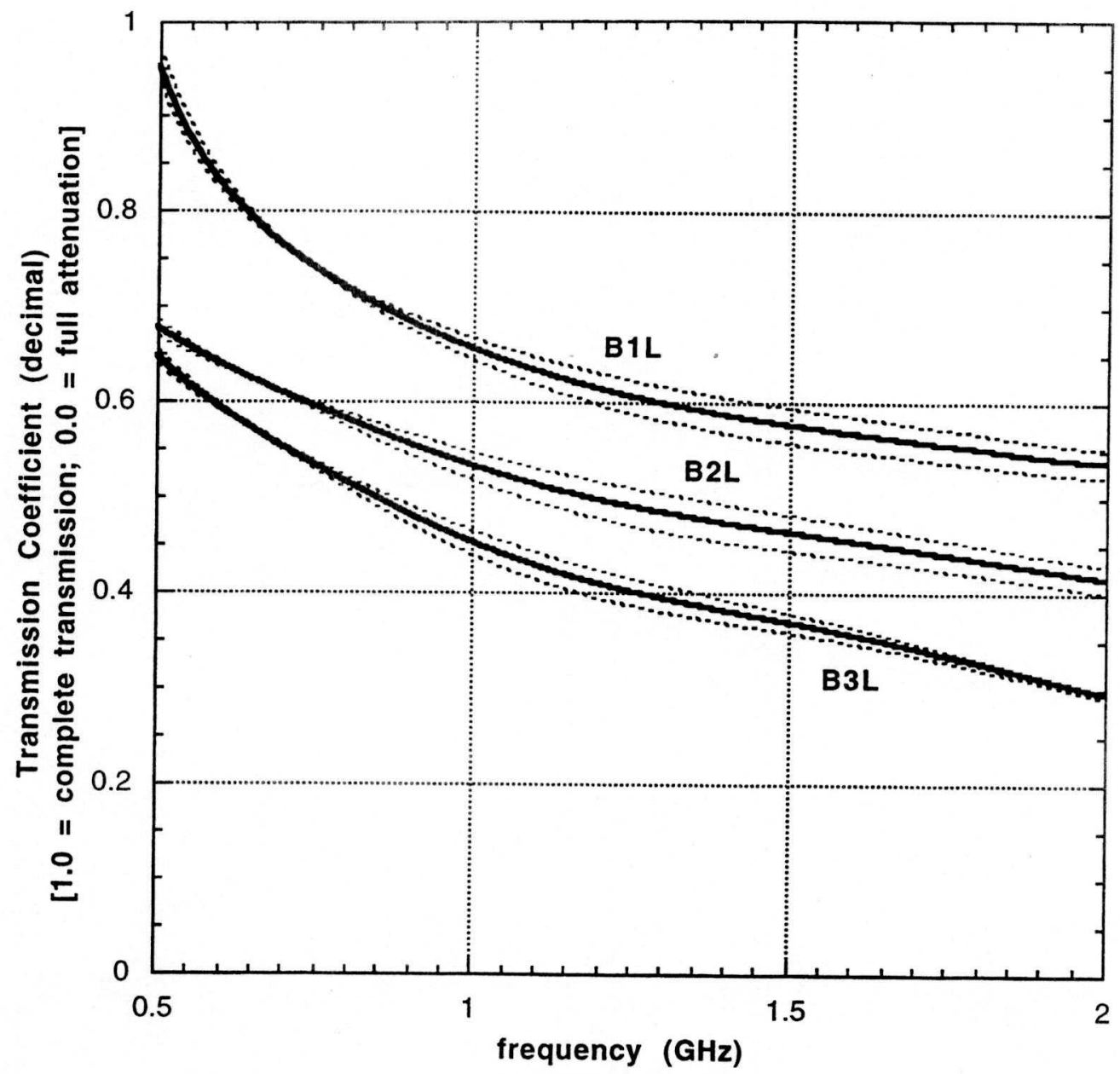

Figure 7.4: Decimal Transmission Coefficients for Brick $B 1 L=89 \mathrm{~mm} ; B 2 L=178 \mathrm{~mm} ; B 3 \mathrm{~L}=267 \mathrm{~mm}$ nominal Target Thickness. Dotted Curves represent $+/$ - 1standard deviation from mean (Solid Curves). Low Range Data: 0.5 to $2.0 \mathrm{GHz}$

concerned, with only minimal power attenuation. Further, because propagation delay times are directly proportional to material density, these latter materials produce smaller deviations from the true distance. It therefore seems likely that NLS technology will first be used for registered-view component placement in residential construction. Its use for precision machine control and component tracking and placement in more cluttered environments will require the use of real-time propagation compensation techniques. These procedures, which include both hardware and software, are being developed at NIST under the aegis of the construction automation and robotics program at the Building and Fire Research Laboratory.

\section{8: Conclusions}

This research project addressed the subject of automated metrology (surveying) for use on construction sites. Specifically, the experimental program has been directed towards the practical development of a novel Non-Line-of-Sight (NLS) survey system with which the real-time position and orientation (attitude) of any object on a construction job site may be determined, irrespective of the presence of intervening obstacles that would otherwise render optical, electro-optical, and other short wavelength electromagnetic distancing techniques useless.

Tests were conducted using a specially configured broadband low frequency spread spectrum radar operating between 0.5 to $8.0 \mathrm{GHz}$. The transmission and receiving antennae, which in normal radar are typical- 
Table 7.1: Regression Coefficients (DECIMAL) for BRICK Transmission versus Frequency Curves Plotted in Figure 4.1a. The regression equation is of the form

Transmission Coefficient $=M 0+M 1 * F+M 2^{*} F^{\wedge} 2+M 3^{\star} F^{\wedge} 3+M 4^{\star} F^{\wedge} 4+M 5^{*} F^{\wedge} 5+M 6^{\star} F^{\wedge} 6$, where $F$ is the frequency in GHz. The standard error term, $R$, is defined in the text.

Data are for the Frequency Range 0.5 to $2.0 \mathrm{GHz}$.

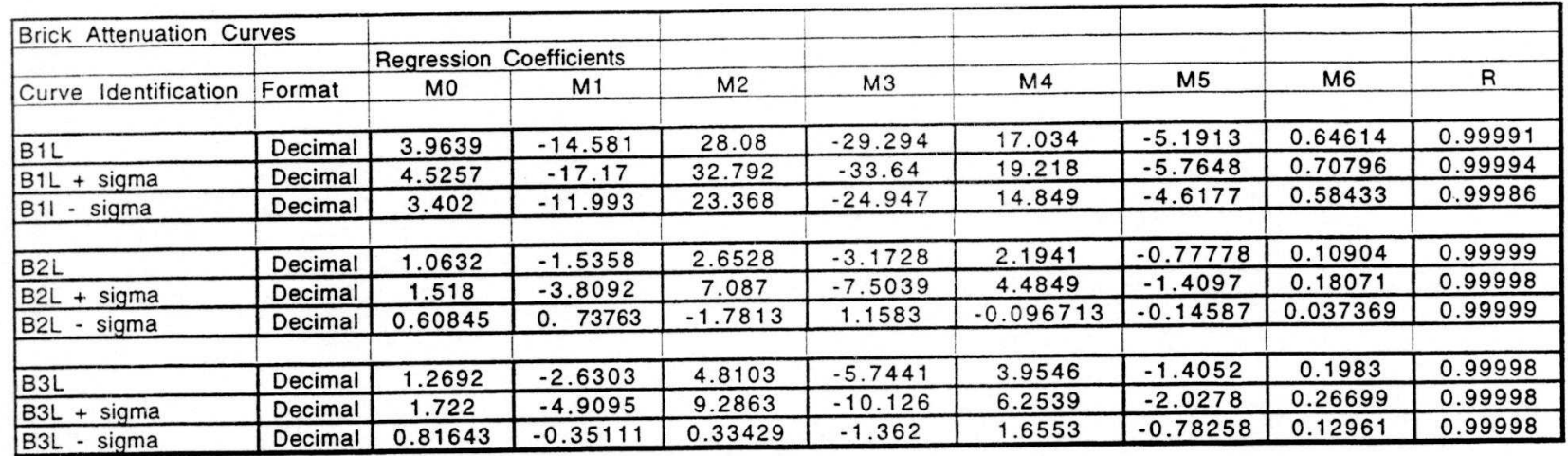

ly one and the same, were physically separated so as to create a system with a fixed broadcast unit and a "roving" receiver, whose range was to be determined relative to the transmission antenna by means of time-ofarrival measurements.

Time domain response was synthesized by means of chirp- $z$ Fourier theory from a broad spectrum of data sampled in the frequency domain. Numerous field and laboratory experiments were performed in which typical construction site obstacles were placed between the transmitter and receiver with separation distances of up to 70 meters in the case of the outdoor tests. The outdoor obstacles included a half-meter thick, heavily reinforced concrete wall, varying combinations of masonry block and brick up to two meters in thickness and at varying angle-of-incidence orientations relative to the transmission path, and metal pre-fabricated wall panels. In all but the latter case, repeatable distance measurements were obtained.

Range detection was lost in the presence of extensive metal panels which contained no windows. However, the presence of even small openings (on the order of several centimeters) permitted range acquisition. Subsequent, extensive, laboratory measurements quantified the behavior of 58 different construction materials involving 1160 tests over two frequency bandwidths $(0.5$ to $2.0 \mathrm{GHz}$ and 3.0 to $8.0 \mathrm{GHz})$. In all cases, unambiguous distance measurements could be acquired. Error sources in the laboratory tests were largely defined by the delay times involved with the propagation of electromagnetic radiation through the construction materials. These errors were highly repeatable, suggesting an avenue towards the development of auto-compensation techniques.
Several types of problems well known to the radar community were observed during the outdoor tests. These included "clutter" (reflections of the transmitted beam off false "targets") and "multipath" (diffracted and scattered elements of the original signal which may, under certain conditions, arrive ahead of the desired signal and which, as a matter of course, may obscure or cast doubt upon which detected signal in the time domain response represents the true transmitter-to-target distance).

The other phenomena, described earlier, is the well known fact that electromagnetic radiation propagates through solid media at velocities less than the speed of light in a vacuum. Thus, any signal transmitted through an engineering material -- e.g. brick, masonry block, or concrete walls -- will appear to have been delayed from its expected arrival time at the receiver. In some cases this delay was sufficient that multipath signals arrived ahead of the "true" signal representing the straight line distance from transmitter to receiver. The delay is directly proportional to the dielectric and the permittivity constants of the engineering material penetrated. Where long distances are involved between the transmitter and receiver the characteristics of the air (including temperature, humidity, and barometric pressure) must be accounted for as well. During the NIST tests this was accomplished automatically by means of a "free space" calibration with no intervening obstacles between the transmitter and receiver at the start of each test series.

Errors due to propagation delays can be significant. Penetration of a half-meter thick outdoor reinforced concrete wall induced a mean range error of 800 $\mathrm{mm}$. For combined masonry block walls faced with 
brick, range errors of three meters were observed for a wall thickness of two meters and a half meter error for a wall thickness of $300 \mathrm{~mm}$. Subsequent extensive laboratory tests showed these range errors to be linearly proportional to the penetration depth (wall thickness) and to the dielectric constant for the material.

While three meters of range error over a $70 \mathrm{~m}$ survey shot is unacceptable for modern construction surveying, it is important to recognize that nearly all of the error is related to propagation delay. The more surprising result is that a measurement through two meters of brick and masonry block was achieved at all! Open field tests [10] have since demonstrated that $10 \mathrm{~mm}$ accuracy or better is achievable using this system. This suggests that it is feasible to develop real-time compensation techniques which will be capable of eliminating errors due to propagation. The development of such a method is currently being investigated at NIST.

\section{8: References}

[1] Stone, W.C., Non-Line-of-Sight (NLS) Metrology, NIST Construction Automation Program Report No. 1, NISTIR 5825, National Institute of Standards and Technology, Gaithersburg, MD, Feb. 1996

[2] Stone, W.C., Ed., Proceedings of the NIST Construction Automation Workshop, March 30-31, 1995, NIST Construction Automation Program Report No. 2, NISTIR 5856, National Institute of Standards and Technology, Gaithersburg, MD, May 1996, pp 1322; 29-42.

[3] Stone, W.C., Electromagnetic Signal Attenuation in Construction Materials, NIST Construction Automation Program Report No. 3, Work in Progress, National Institute of Standards and Technology, Gaithersburg, MD.

[4] Ulaby, F.T., Whitt, M., and Sarabandi, K., AVNA Based Polarimetric Scatterometers, IEEE Antennas and Propagation Magazine, October 1990, pp. 6-17.

[5] Blejer, D.J., Frost, C.E., and Scarborough, S.M., Theory and Measurements of Buried and Partially Buried Trihedrals for Ground Penetration Radar, Project Report GPR-3, MIT Lincoln Lab, 22 February 1995.

[6]Churchill, R., Complex Variables and Applications, McGraw-Hill, 1984.
[7] Rabiner, L.R., and Gold, B., Theory and Application of Digital Signal Processing, Prentice-Hall, 1975, pp. 356-399.

[8]Krauss, T.P., Shure, L., and Little, J.N., Signal Processing Toolbox, The Math Works, Inc., Natick, MA, September 1995. pp. 1.56-1.57.

[9] HP 8530A Microwave Receiver Operating Manual, HP Part No. 08350-90010, February 1993, Edition 2, pp. $13.12-13.14$.

[10] Scarborough, S, Frost, C, and Blejer, D., Range Accuracy Experiment, Letter Report from MIT Lincoln Lab to NIST, November 1, 1996.

* Any mention of commercial products is for information only; it does not imply recommendation or endorsement by the National Institute of Standards and Technology nor does it imply that the products mentioned are necessarily the best available for the purpose. 